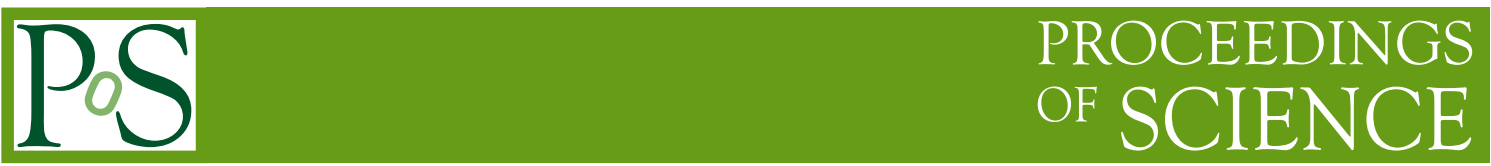

\title{
Detection of virial shocks in stacked Fermi-LAT clusters
}

\author{
Ido Reiss* \\ Physics Department, Ben-Gurion University of the Negev, Israel \\ Physics Department, Nuclear Research Center Negev, Israel \\ E-mail: reissi@post.bgu.ac.il

\section{Jonathan Mushkin} \\ Physics Department, Ben-Gurion University of the Negev, Israel

\section{Uri Keshet} \\ Physics Department, Ben-Gurion University of the Negev, Israel \\ E-mail: ukeshetabgu.ac.il
}

\begin{abstract}
In the hierarchical paradigm of structure formation, galaxy clusters are the largest objects ever to virialize. They are thought to grow by accreting mass through large scale, strong virial shocks. Such a collisionless shock is expected to accelerate relativistic electrons, thus generating a spectrally flat leptonic virial ring. However attempts to detect virial rings have all failed, leaving the shock paradigm unconfirmed. Here we identify a virial $\gamma$-ray signal by stacking Fermi-LAT data for 112 clusters, enhancing the ring sensitivity by rescaling clusters to their virial radii and utilizing the anticipated spectrum. In addition to a central unresolved, hard signal (detected at the nominal $5.8 \sigma$ confidence level), probably dominated by active galactic nuclei, we identify $(5.9 \sigma)$ a bright, spectrally flat $\gamma$-ray ring at the expected shock position. It corresponds to $\sim 0.6 \%$ (with an uncertainty factor $\sim 2$ ) thermal energy deposition in relativistic electrons over a Hubble time. This result validates the shock paradigm, calibrates its parameters, and indicates that the cumulative emission from such shocks significantly contributes to the diffuse extragalactic $\gamma$-ray and radio backgrounds.
\end{abstract}

7th Fermi Symposium 2017

15-20 October 2017

Garmisch-Partenkirchen, Germany

${ }^{*}$ Speaker. 